\title{
Evolution de la Pression Glossinaire à la Station d'Avétonou au Sud-Ouest du Togo
}

\author{
Kotoe Mensah Délako,
}

Institut Togolais de Recherche Agronomique ; Centre de recherche Agronomique-zone Forestière (ITRA/CRAF) Station Avétonou, Kpalimé

\section{Seme Kpassi}

Institut Togolais de Recherche Agronomique ; Centre de recherche Agronomique-zone Forestière (ITRA/CRAF) Station Avétonou, Kpalimé Ecole Supérieure d'Agronomie, Département des Sciences Animales et

Vétérinaires, Université de Lomé ESA/UL, Lomé, Togo

\section{Kossoga Kakom Assota, Koumessi Léopold Komi,}

Institut Togolais de Recherche Agronomique ; Centre de recherche Agronomique-zone Forestière (ITRA/CRAF) Station Avétonou, Kpalimé Dao Badabadi ${ }^{2}$;

RACH Liaison Officer to WAHO and RCDSC ; Regional Animal Health Center ; Campus of Sotuba Bamako, Mali

\section{Talaki Essossina, Pitala Wéré,}

Ecole Supérieure d'Agronomie, Département des Sciences Animales et Vétérinaires, Université de Lomé ESA/UL, Lomé, Togo

\section{Lombo Yao,} Kpemoi Kossi,

Direction Générale de l'Institut Togolais de la Recherche Agronomique

(ITRA) Lomé-Togo

\section{Résumé}

La station d'Avétonou constitue un site de réservoir de vecteur de glossine à cause de sa faune sauvage importante et son biotope favorable car elle est proche du fleuve Zio et jouit d'un climat relativement humide et d'une végétation constituée de forêt galerie et de savane arborée. Aussi, la prévalence de la maladie dans les troupeaux ne cesse de croître d'année en année et l'élevage bovin dans la zone n'a cessé d'enregistrer des difficultés d'ordre pathologique et économique liées à la prolifération et aux menaces des glossines dans le milieu. Cette étude a pour objectif de faire l'état des lieux et 
actualiser les résultats de recherche jadis acquis dans les années 1980 par Mawuena et reprise en 2011 par Talaki afin de rechercher dans l'avenir une approche de solution à ce problème. Pour se faire, des enquêtes entomologiques et parasitologiques ont été menées en 2016 à la station d'Avétonou et dans les villages environnants. Les résultats ont montré que la densité moyenne des glossines est passée de 0,2 (1986) à 2,2 (2011) puis à 1,06 (2016) soit une augmentation de plus de cinq fois. Dans le même ordre, la prévalence de la maladie est passée de $4,7 \%$ à $22,78 \%$ puis à $23,8 \%$. Trypanosoma vivax et Glossina palpalis palpalis ont été les espèces de parasites et de vecteurs les plus dominants. Cette évolution sans cesse croissante de la prolifération des glossines et de son infestation risque de compromettre l'élevage bovin dans le milieu; des mesures de lutte contre le vecteur doivent être entreprises pour préserver la santé des troupeaux et sauvegarder l'élevage des bovins en général dans la zone.

Mots-clés : Parasites, Prévalence, Tolérance, Infestation, Bovins, Trypanosomose 


\title{
Evolution of Tsetse Pressure at Avetonou Station in the South-West of Togo
}

\author{
Kotoe Mensah Délako,
}

Institut Togolais de Recherche Agronomique ; Centre de recherche

Agronomique-zone Forestière (ITRA/CRAF) Station Avétonou, Kpalimé

\section{Seme Kpassi}

Institut Togolais de Recherche Agronomique ; Centre de recherche Agronomique-zone Forestière (ITRA/CRAF) Station Avétonou, Kpalimé Ecole Supérieure d'Agronomie, Département des Sciences Animales et

Vétérinaires, Université de Lomé ESA/UL, Lomé, Togo

\section{Kossoga Kakom Assota, Koumessi Léopold Komi,}

Institut Togolais de Recherche Agronomique ; Centre de recherche Agronomique-zone Forestière (ITRA/CRAF) Station Avétonou, Kpalimé Dao Badabadi ${ }^{2}$;

RACH Liaison Officer to WAHO and RCDSC ; Regional Animal Health Center ; Campus of Sotuba Bamako, Mali

\section{Talaki Essossina, Pitala Wéré,}

Ecole Supérieure d'Agronomie, Département des Sciences Animales et Vétérinaires, Université de Lomé ESA/UL, Lomé, Togo

\section{Lombo Yao,} Kpemoi Kossi,

Direction Générale de l'Institut Togolais de la Recherche Agronomique

(ITRA) Lomé-Togo

\begin{abstract}
The Avetonou station is one of the oldest animal production research stations in the sub-region of West Africa. The new directions of the station in recent years no longer allow the continuation and the sustainable achievements of research, especially in the fight against the animal trypanosomiasis undertaken since the 1980s. Also, it is necessary to point out that since then, cattle breeding in the area have not stopped recording pathological and economical difficulties due to the proliferation and threats of the tsetse in the area. This paper, however, focuses on highlighting the point and updating the research results previously acquired in the 1980 s by Mawuena and recovered
\end{abstract}


in 2011 by Talaki. This is with the aim of seeking a solution approach in addressing this problem in the future. To do this, entomological and parasitological surveys were conducted in 2016 in the station of Avetonou and in the surrounding villages. The results showed that the average density of the tsetse went from 0.2 (1986) to 2.2 (2011), and then to 1.06 (2016) resulting in an increment of more than five times. Similarly, the prevalence of the disease rose from $4.7 \%$ to $22.78 \%$, and then to $23.8 \%$. Trypanosoma Vivax and Glossina palpalis palpalis were the most dominant species of parasites and the vectors. The wooded vegetation of the station, the demographic pressure on the flora, and the proliferation of wild animals might be the reasons for this significant infestation.

Keywords: Parasites, Prevalence, Tolerance, Infestation, Cattle, Trypanosomiasis

\section{Introduction}

La trypanosomose constitue l'une des principales maladies animales au Togo à l'instar des autres pays au Sud du Sahara (Pregrine, 1994 ; Swallow, 2000). Le vecteur principal est la mouche tsé-tsé et son gîte préférentiel se retrouve surtout dans les zones à forte densité animale et à végétation arborée en zone humide (Dial, 2001). Plusieurs études ont été conduites au Togo notamment à la station d'Avétonou dans les années 1980 et en 2011 en vue de leur éradication (Mawuena, 1987 ; Talaki, 2013). Dès lors, la prolifération du vecteur dans la zone d'Avétonou n'a cessé de s'accroître eu égard aux nombreux cas de pathologies bovines signalées dues à ces vecteurs. En effet, la station d'Avétonou constitue un site de réservoir du vecteur à cause de sa faune sauvage importante et son biotope favorable car elle est proche du fleuve Zio et jouissant d'un climat relativement humide et une végétation constituée de forêt galerie et de savane arborée. Aussi, la prévalence de la maladie dans les troupeaux ne cesse -t-elle de croître d'année en année. En effet, elle est passée de 21\% (2008) à 23\% (2009) à 25\% (2010) (ITRA, 2010) à 22,78\% (2011) (Talaki, 2013). Les pertes économiques engendrées sont importantes : la mortalité des animaux, les faibles poids des animaux par rapport à leurs âges, la faible productivité du cheptel, le coût élevé des frais sanitaires, etc. (Macleannan, 1980 ; ITRA, 2005 ; Talaki, 2013). Il serait d'autre part judicieux de lier la recrudescence de la maladie aux changements climatiques et aux pressions démographiques sur l'écosystème observé ces dernières années (Talaki, 2013). En effet, les périodes de pluie et de sécheresse ne sont plus respectées et les surfaces cultivables des agriculteurs se sont redoublées à cause de la croissance démographique. Les anomalies climatiques ont profondément modifié les habitudes agropastorales et le calendrier cultural n’est plus maitrisé. Ce désordre écologique a des répercussions sur la faune et 
la flore et a occasionné des mouvements migratoires de la faune sauvage et des glossines d'une zone à une autre. La présente étude a pour but de faire l'état des lieux et actualiser les données de l'infestation glossinaire du milieu après les études de Mawuena dans les années 1980 et celle de Talaki en 2011 et mesurer son impact sur l'état sanitaire du bétail.

\section{Matériel et méthodes}

\subsection{Zone d'étude}

L'étude s'est déroulée à la station de recherche zootechnique et vétérinaire d'Avétonou. En effet, la station a été créée depuis 1963 dans le cadre du projet de la coopération Germano-togolaise pour la promotion de l'élevage du bétail trypanotolérant au Togo. Elle est située à $100 \mathrm{~km}$ de Lomé (capitale du Togo), au Sud-Ouest entre 640' et 6 $6^{\circ} 55^{\prime}$ latitude Nord, 040'et $0^{\circ} 55$ longitude Est. Les monts Agou, à $5 \mathrm{~km}$ plus au nord constitue les massifs montagneux prédominants avec point culminant $986 \mathrm{~m}$. Le fleuve Zio qui coule du Nord vers le Sud-Est, situé à $2 \mathrm{~km}$ de la station, demeure le cours d'eau le plus important. La station bénéficie d'un climat de type soudanoguinéen avec une grande saison sèche qui s'étend de novembre à février et une petite en juillet - août; deux saisons pluvieuses qui s'étendent de mars à juin et en septembre -octobre. La pluviométrie moyenne annuelle varie entre 1100 et $1400 \mathrm{~mm}$ avec les plus grandes précipitations en juin. La végétation est constituée par un ensemble de savane arborée traversée par endroit de forêts galeries. La faune sauvage est constituée d'antilopes, de biches, de phacochères, de rongeurs, de reptiles, etc. (Mawuena et al. 1981).

\section{2. Matériel et animaux utilisés}

Quarante (40) pièges biconiques ont été utilisés pour la capture des glossines. (Mihok, 2002) Des tubes vacutainers munis d'anticoagulant (héparines) ont servi pour les prélèvements sanguins.

Cent quatre-vingt-un (181) bovins des deux sexes, de race N'Dama, et métis (zébu x N'dama ; zébu x taurins) ont été utilisés pour les enquêtes parasitologiques. La rive du fleuve Zio (site A), la station d'Avétonou (site B) et les villages environnants (site C) ont servi de zone d'expérience (Figure 1). 


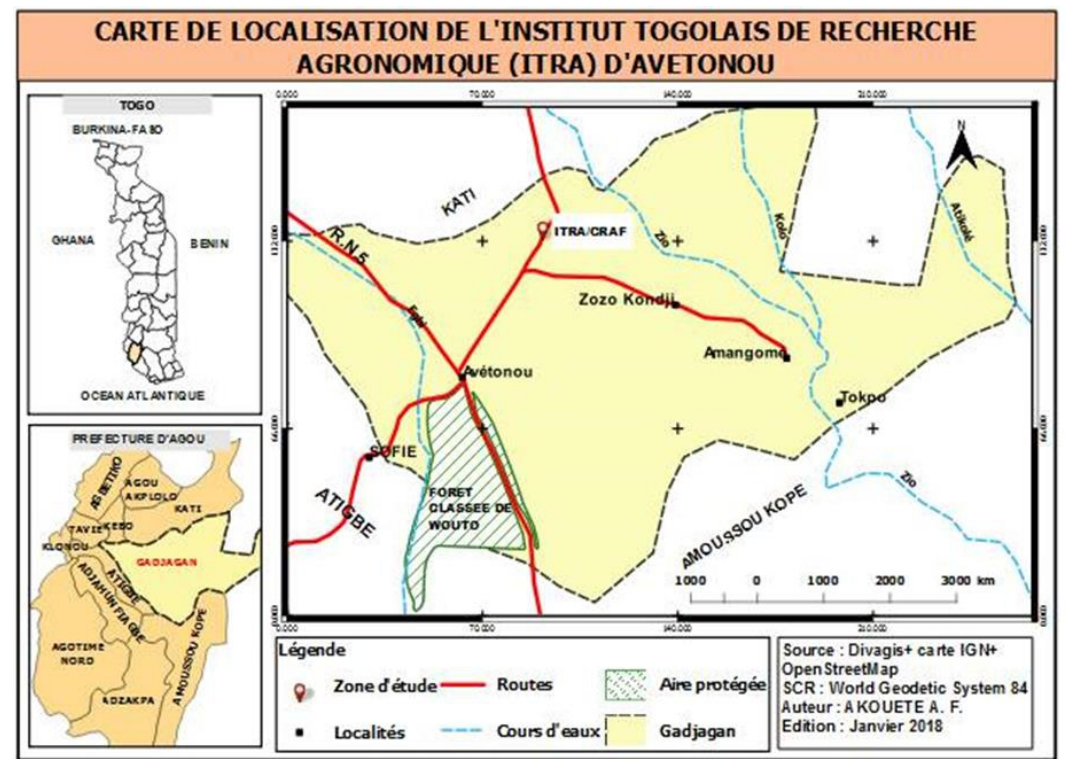

Figure 1 : Carte du Togo indiquant la station d'Avétonou de l'ITRA/CRAF

\subsection{Méthodes entomologiques et parasitologiques utilisées}

\subsubsection{Méthode entomologique}

L'expérience a débuté en août 2015 et s'est achevée en février 2016. Des pièges ont été placés dans l'espace de la station, aux points d'abreuvement des troupeaux, dans les couloirs d'acheminement des animaux, dans les villages environnants et dans les forêts galeries. Les pièges sont espacés de $100 \mathrm{~m}$ (Lekeux, 2006) et les récoltes ont été faites par fréquence de deux jours consécutifs au cours de chaque mois. Les glossines capturées sont examinés au laboratoire de la station pour l'indentification. Les paramètres recherchés ont été l'espèce et la densité apparente des glossines (DAG) par la formule (1) de Leak et al. (1984). Les mouvements migratoires des glossines ont été aussi étudiés suivant les trois saisons qui ont été déterminées en fonction de la pluviométrie enregistrée au cours de la période de l'expérience. Il s'agit de la saison humide (février et août), la saison des pluies (septembre et octobre) et saison chaude (novembre, janvier).

Densité apparente des glossines $(D A G)=$ nombre moyen de mouches prises par piège et par jour (1)

\subsubsection{Méthode parasitologique}

Le contrôle de l'infestation trypanosomienne a été évalué après prélèvement sanguin au niveau de la veine jugulaire suivi d'examen par la technique de micro centrifugation en tube capillaire selon la méthode de Buffy Coat décrite par Murray et al. (1990). Le degré d'anémie des animaux a été aussi étudié par la détermination de l'hématocrite, la valeur du PCV (Packed 
Cells Volume). Le PCV de niveau 25 a été retenu comme seuil d'indicateur d'un état anémique ( Lekeux 2006 ; Talaki et al. 2013).

\subsection{Analyse des données}

Les résultats ont été exprimés en moyenne plus ou moins écart type. Les différentes moyennes ont été comparées par l'analyse de variance à l'aide des tests $\mathrm{F}$ et de Student. Une analyse des données quantitatives de la pluviométrie et de la densité des glossines a été faite à l'aide du logiciel XLSTAT version 7.1. Le coefficient de corrélation entre les différentes variables des densités et la pluviométrie a été déterminé.

\section{Résultats}

\subsection{Entomologie}

Les mouches tsé-tsé Glossina tachinö̈des et Glossina palpalis palpalis ont été les espèces piégées avec les densités moyennes de 1,06 pour l'ensemble des trois sites avec une dominance des G. tachinoïdes (Tableau 1). Tableau 1 : Espèces de glossines rencontrées et leur densité

\begin{tabular}{llll}
\hline Sites & \multicolumn{2}{c}{ Glossines } & Total \\
\cline { 2 - 4 } & G. tachinoïdes & G. palpalis palpalis \\
\cline { 2 - 4 } $\mathrm{A}$ & 0,56 & 0,08 & 0,64 \\
$\mathrm{~B}$ & 0,89 & 0,02 & 0,91 \\
$\mathrm{C}$ & 1,62 & 0,02 & 1,64 \\
\hline Moyenne & 1,03 & 0,04 & 1,06 \\
\hline
\end{tabular}

La fluctuation mensuelle des glossines a une densité supérieure à 1 (DAG >1) d'août à octobre et inférieur à $1(\mathrm{DAG}<1)$ de novembre à février (Tableau 2).

Tableau 2. Fluctuation mensuelle des glossines et leur densité

\begin{tabular}{lllllllll}
\hline Zones & \multicolumn{9}{c}{ Mois } & & Moyenne \\
\cline { 2 - 9 } & Août & Sept & Oct & Nov & Déc & Janv & Fév & \\
\cline { 2 - 9 } A & 0,22 & 1,25 & 1,40 & 0,90 & 0 & 0 & 0,08 & 0,64 \\
B & 1,43 & 1,73 & 0,85 & 0,70 & 0 & 0,29 & 0,46 & 0,91 \\
C & 1,17 & 1,39 & 4,60 & 0 & 0,10 & 0,25 & 2,40 & 1,64 \\
\hline Moyenne & 1,01 & 1,46 & 2,28 & 0,58 & 0,03 & 0,18 & 0,98 & 1,06 \\
\hline
\end{tabular}

Les plus fortes densités de glossine sont enregistrées dans les mois d'août à octobre.

-Evolution glossinaire des années 1977 à 2016 à la station et ses environs

La situation des glossines de 2016 comparée à celle des années 1977 ; 1987 et 2011 montre que G. palpalis palpalis sont en disparition au profit de G. tachnoïdes (Tableau 3). 
Tableau 3 : Densité des glossines de 2016 comparée à celle des années 1977 ; 1987 et 2011

\begin{tabular}{lllll|llll}
\hline Zone & \multicolumn{4}{c}{ G. tachinoüdes } & \multicolumn{3}{c}{ G. palpalis palpalis } \\
\cline { 2 - 9 } & $\mathbf{1 9 7 7}$ & $\mathbf{1 9 8 7}$ & $\mathbf{2 0 1 1}$ & $\mathbf{2 0 1 6}$ & $\mathbf{1 9 7 7}$ & $\mathbf{1 9 8 7}$ & $\mathbf{2 0 1 1}$ & $\mathbf{2 0 1 6}$ \\
$\mathrm{A}$ & 2,00 & 0,20 & 0,33 & 0,56 & 4,00 & 0,80 & 1,67 & 0,08 \\
$\mathrm{~B}$ & 0,60 & 0,05 & 2,5 & 0,89 & 1,00 & 0,10 & - & 0,02 \\
$\mathrm{C}$ & 0,05 & - & 2 & 1,62 & - & - & & 0,02 \\
\hline Moyenne & $\mathbf{0 , 8 8}$ & $\mathbf{0 , 1 3}$ & $\mathbf{2}$ & $\mathbf{1 , 0 2}$ & $\mathbf{2 , 5}$ & $\mathbf{0 , 4 5}$ & $\mathbf{0 , 2}$ & $\mathbf{0 , 0 4}$ \\
\hline
\end{tabular}

L'évolution de la densité des glossines en fonction de la saison et de la pluviométrie montre qu'elle est plus élevée en saison de pluie et presque nulle en saison chaude (Tableau 4). De plus, il existe une corrélation significative $(p \leq 0,05)$ entre la pression glossinaire et la pluviométrie avec un coefficient de variation de 0,955 (Figure 1).

Tableau 4 : Densité des glossines suivant les saisons de la zone d'étude

Mois

\begin{tabular}{|c|c|c|c|c|c|c|c|}
\hline & Août & Septembre & Octobre & Novembre & Décembre & Janvier & Février \\
\hline Saisons & $\begin{array}{l}\text { Saison } \\
\text { humide }\end{array}$ & Saison de & pluie & Sais & n Chaude & & $\begin{array}{l}\text { Saison } \\
\text { humide }\end{array}$ \\
\hline $\begin{array}{l}\text { Pluviométrie } \\
(\mathbf{m m})\end{array}$ & 57 & 195 & 252 & 0 & 0 & 0 & 84 \\
\hline Densité & 1,01 & 1,46 & 2,28 & 0,58 & 0 & 0,18 & 0,98 \\
\hline
\end{tabular}

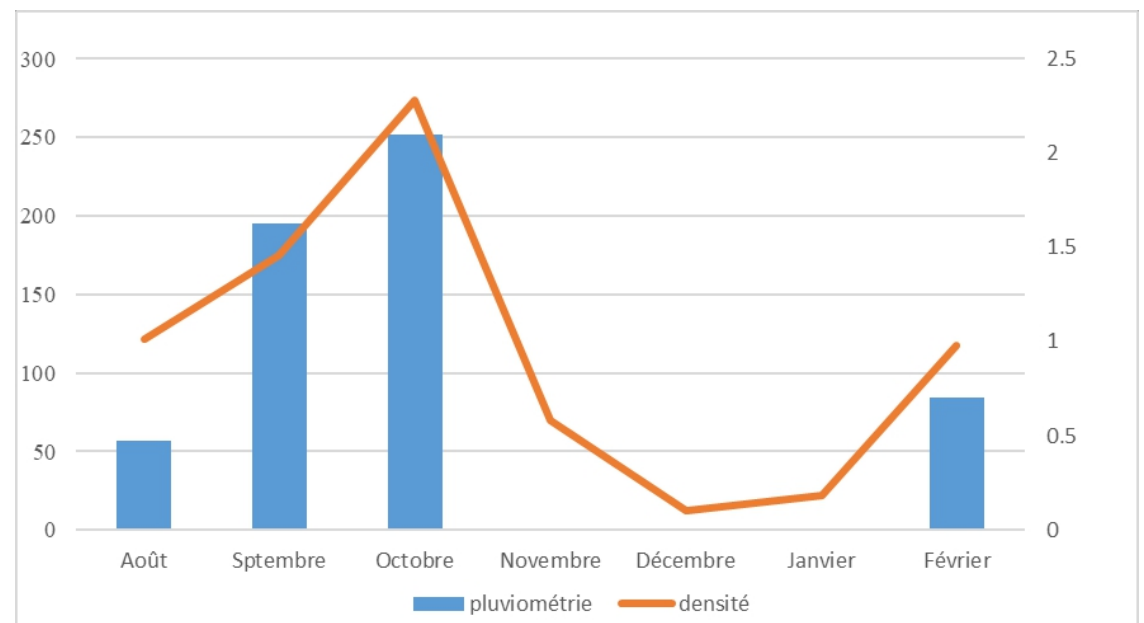

Figure 2 : Evolution mensuelle des glossines en fonction de la pluviométrie et de la densité

\subsection{Parasitologie}

Les parasites retrouvés dans le sang ont été $T$. congolensis $(3,9 \%)$ et T. vivax $(19,9 \%)$. Toutes les races ont été sensibles aux deux parasites avec une forte prévalence chez les métis $(30,2 \%)$ (Tableau 5). 
Tableau 5 : Prévalence des bovins à l'espèce de trypanosome

\begin{tabular}{|c|c|c|c|c|c|c|}
\hline \multirow[b]{2}{*}{ Races } & \multicolumn{2}{|c|}{ T. vivax } & \multicolumn{2}{|c|}{ T. congolensis } & \multirow[b]{2}{*}{$\begin{array}{l}\text { Total } \\
\text { infestation }\end{array}$} & \multirow{2}{*}{$\begin{array}{l}\text { Prévalenc } \\
\text { e } \\
(\%)\end{array}$} \\
\hline & $\begin{array}{l}\text { Cas } \\
\text { positif }\end{array}$ & Prévalence $(\%)$ & Cas positif & $\begin{array}{l}\text { Prévalence } \\
(\%)\end{array}$ & & \\
\hline N'Dama $(n=68)$ & 9 & 13,23 & 3 & 4,41 & 12 & 17,63 \\
\hline Locale $(n=60)$ & 12 & 20 & 3 & 5 & 15 & 25 \\
\hline Métis $(n=53)$ & 15 & 28,30 & 1 & 1,90 & 16 & 30,2 \\
\hline Total $(n=181)$ & 36 & 19,9 & 7 & 3,9 & 43 & 23,8 \\
\hline
\end{tabular}

\subsection{Etat d'anémie des bovins (PCV)}

Par race, les N'Dama $(89,7 \%)$; les métis $(67,65 \%)$ et les locales $(60 \%)$ ont connu un $\mathrm{PCV} \geq 25$ (Tableau 6)

Tableau 6 : Le PCV en fonction des races

\begin{tabular}{|c|c|c|c|c|c|c|}
\hline \multirow[b]{2}{*}{ Race } & \multicolumn{3}{|l|}{$\mathrm{PCV}<25$} & \multicolumn{3}{|c|}{$\mathrm{PCV} \geq 25$} \\
\hline & $\begin{array}{l}\text { Nombre } \\
\text { de cas }\end{array}$ & $\%$ & $\begin{array}{l}\text { Ecart } \\
\text { type }\end{array}$ & $\begin{array}{l}\text { Nombre } \\
\text { de cas }\end{array}$ & $\%$ & $\begin{array}{l}\text { Ecart } \\
\text { type }\end{array}$ \\
\hline $\begin{array}{l}\text { N'Dama } \\
\text { N=58 }\end{array}$ & 6 & 10,3 & 6 & 52 & 89,7 & 5 \\
\hline $\begin{array}{l}\text { Locale } \\
\mathrm{N}=50\end{array}$ & 20 & 40 & 20 & 30 & 60 & 4 \\
\hline $\begin{array}{l}\text { Métisse } \\
\mathrm{N}=68\end{array}$ & 22 & 32,5 & 22 & 46 & 67,65 & 5 \\
\hline
\end{tabular}

Dans chaque troupeau, plus de $50 \%$ des animaux ont un $\mathrm{PCV} \geq 25$.

\section{Discussion}

\subsection{Entomologie}

Après trente ans (de 1986 à 2016) de travaux sur la trypanosomose et son vecteur à la station d'Avétonou, la densité des glossines est passée de 0,2 à 1,06 soit une augmentation de $53,75 \%$. Parmi cette population, l'espèce $G$. tachinoïdes a été la plus dominante $(\mathrm{DAG}=1,02)$ contre l'espèce $\mathrm{G}$. palpalis palpalis (DAG $=0,04)$. Ces résultats corroborent aux études de Talaki (2013) en 2011 et de Hendrickx et al. (1999) ayant obtenu respectivement DAG =2 pour $G$. tachinoides contre 0,2 pour $G$. palpalis palpalis. Alors que les travaux antérieurs de Mawuena (1987) avait démontré que G. palpalis palpalis était la plus importante $(\mathrm{DAG}=2,5)$ contre $\mathrm{G}$. tachinoïdes $(\mathrm{DAG}=0,88)$ dans les années 1977. Cet inversement de situation serait imputable à la fois aux aléas climatiques et à la destruction du biotope naturel de l'espèce suite à la pression démographique sur la flore pour des activités agricoles dans le site de la rive de Zio. Ainsi, G. palpalis palpalis est en voie de disparition (Magalie, 2006) au profit de G. tachinö̈des qui, lui a tendance à émigrer vers la station où se trouve un gîte plus favorable (végétation de plus en plus arborée, pratique d'élevage sédentaire, présence d'une faune sauvage abondante, absence 
d'activités agricoles). Talaki (2013) a rapporté que seule l'espèce $G$. tachinoïdes a été capturée dans la zone d'étude avec un $\mathrm{DAP}=2$ contre un $\mathrm{DAP}=0,2$ de G.palpalis palpalis qui n'a été capturée que dans la rive du fleuve Zio. Les mouvements migratoires de ces espèces ont lieu surtout en période sèche. En effet, les densités plus fortes ( $\mathrm{DAG}=1,46$ et 2,28) et les plus faibles (DAG $=0,58$ et 0,18 ) ont été enregistrées respectivement lorsque les pluviométries ont atteint leur maximum (195-252 $\mathrm{mm}$ ) et leur minimum (traces) de pluie. Itard (1986) a démontré que c'est durant la saison des pluies qu'à lieu une explosion démographique de population de glossine. La présence des G. tachinoïdes dans la zone d'Avétonou est inhabituelle car selon les travaux de Rickenbach (1961), et de Itard (1968) G. tachnoïdes se limite au Nord au $14^{\mathrm{è}}$ parallèle du Mali et du Burkina Faso et sa limite Sud se situerait au $9^{\circ} \mathrm{C}$ Nord pour le Togo. Avétonou se situe à la $6^{\circ} \mathrm{C}$ latitude Nord, la présence de ce vecteur dans la zone nous laisse croire que $G$. tachinoüdes aurait progressé plus au Sud que sa limite présumée (Mawuena, 1980). La présente étude confirme les travaux de cet auteur cependant avec une densité plus importante 1,03 contre 0,05 .

\subsection{Parasitologie}

La $T$. vivax et la $T$. congolensis ont été les espèces de trypanosome bovin rencontré à la station d'Avétonou et ses environs avec une prévalence de $23,80 \%$ dont $19,9 \%$ pour $T$. vivax et $3,9 \%$ pour le $T$. congolensis. Cette prévalence est supérieure à celle observée par Leak (1986) (4,7\%), Mawuena (1987) (13,5\%) et celle de Talaki (2013) (22,78\%) à la station d'Avétonou. Cette prévalence à la trypanosomose a été plus remarquable chez les métisses $(30,20 \%)$ et plus faible chez les N'Dama $(17,63 \%)$. La diminution relative de la prévalence chez les N'Dama proviendrait du facteur génétique de la trypanotolérence qui est observée chez cette race (FAO, 1979). Par contre, chez les métis, cette sensibilité est plus prononcée car ils sont issus du croisement zébu x taurins. Itard (1981), et Touré (1977) ont montré que la tolérance chez les races métisses (croisement entre les races taurines et zébu) connait une limite. Aussi, l'indicateur de l'anémie (PCV) qui matérialise le concept de la trypanotolérence, a montré que, plus de la moitié des animaux toute race confondue a connu un PCV supérieur à la moyenne classique $(\mathrm{PCV} \geq 25)$ malgré la forte pression parasitaire (Murray et al. 1988 ; Talaki et al. 2013). Sur cette base, nous estimons que la majorité des animaux de la station sont tolérants à la trypanosomose. Cette trypanotolérence serait due : (i) à la prédominance du parasite de l'espèce $T$. vivax $(72 \%)$ sur l'espèce $T$. congolensis $(14 \%)$ à la station; (ii) à la faible pathogénicité de $T$. vivax par rapport à $T$. congolensis (Fimmen et al. 1980); (iii) à la conservation de la résistance du $T$. vivax (16 mois) au cours du cycle biologique chez la glossine (Mainguet, 2000 ; Gray et al. 1971). Par ailleurs, nous pensons que l'espèce 
dominante (T. vivax) a été provoquée par le vecteur G. tachinoüdes, étant donné la supériorité en densité par rapport au G. p. palpalis dans le milieu (Dao et al. 2008). Cependant, Leak et al. (1987) ont suggéré qu'en raison de la forte prolifération des G. p. palpalis à la station, 92\% de l'infestation au $T$. vivax sont provoquées par ces dernières. Au vu de ce constat, nous pouvons dire que l'espèce $T$. vivax peut être transmise à la fois par les deux vecteurs $G$. tachinö̈des et G. palpalis palpalis (Megalie, 2006 ; Mihok et al. 1995).

\section{Conclusion}

Après plusieurs années de travaux, la densité des glossines a augmenté de $53,75 \%$ et la prévalence de la maladie a été multipliée par cinq dans la zone d'Avétonou. Malgré cette forte prévalence, la majorité des animaux ont acquis une tolérance vis-à-vis du trypanosome qui aurait été provoquée par l'espèce T. vivax. Par ailleurs, la glossine, de l'espèce G. palpalis palpalis est en voie disparition au profit de G. tachinö̈des ; cette dernière qui pourtant avait sa limite au $9^{\circ}$ Nord du Togo, se localise désormais plus au Sud au-delà du $6^{\circ}$ Nord. Le changement climatique et la pression démographique seraient les principales causes de ces mouvements migratoires. Des dispositions doivent être prises pour lutter contre la migration et la prolifération de ces vecteurs afin de sauvegarder l'élevage bovin dans le milieu.

\section{Remerciements}

Les auteurs de cet article tiennent à remercier l'Institut Togolais de Recherche Agronomique (ITRA) et le Centre de Recherche Agronomique Zone Forestière (CRA-F) pour leur soutien matériels et financier, les techniciens de la station d'Avétonou et les bouviers pour leur acceptation et leur disponibilité pour le succès de ce travail.

\section{References:}

1. Challier A., Lavoisier C., 1973, Un nouveau piège pour la capture des glossines (Glossina : Diptera, Muscidae) : description et essai sur terrain, cahier ORSTOM, série Entomologie médicale et parasitologie, 10 (4) : 251-262.

2. Dao B., Sidibé I., Hendrickx G., Belem A.M.G., et De La Roque, 2008, Impact de la sécheresse et la dégradation des aires protégées sur la répartition des trypnosomoses bovines et leurs vecteurs dans le bassin versant de l'ôti au nord du Togo. Revue d'élevage et de médecine vétérinaire des pays tropicaux, 61 (34) : 153-160.

3. Dial O., 2001. Utilisation des trypanocides au Mali : Facteurs de risque de chimiorésistance. In : Utilisation des trypanocides en Afrique subsahérienne. Actes de séminaire sous régional. Dakar, EISMV, du 06 au 08 Fev. 2001, 125-130. 
4. Gray, A.R. Roberto, C.J., 1971, The stability of resistence of dinamizene aceturate and quinapyramine sulphate in a strain of trypanosoma vivax during cyclical transmission through antilop. Parasitology, 63, 163-168

5. Fimmen H., Dillmann S., Dovie A., 1980, Pathogenéité de deux espèces de glossine : G. congolensis et G. brucéi chez les métis croisés à Avétonou. Trypanotolérence et production animale No 1.

6. Hendrickx,G. et Napala A.,1999, Le contrôle de la trypanosomose < à la carte>:une approche intégrale basée sur un système d'information géographique. Académie Royale des Sciences d'Outre-Mer, classe des

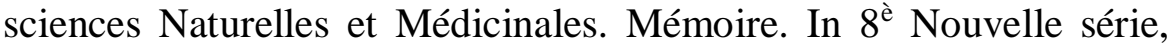
tome 24, fasc. 4, Bruxelles.

7. d'Ieteren,G.D.M., Awuome K., Bokovi K., Shema S., 1987,Facteurs génétiques et de l'environnement affectant la prévalence trypanosomienne et la parasitémie chez le bétail. In Production animale dans les régions d'Afrique infestées par les glossines. Compte rendu de réunion 23-27 novembre 1987 Nairobi. Kénya. In : Production animale dans les régions d'Afrique infestée par les glossines. Nairobi (Kenya), CIPEA/ILRAD 1988.

8. FAO/ILCA/UNE P., 1979, Trypanotolerant livestock in West and central Africa. Monograph No. 2 International Livestock Centre for Africa : Addis Ababa

9. Itard, J., 1981, Trypanosomes animales africaines ; deuxième partie du précis de parasitologie vétérinaire tropicale $773 \mathrm{pp}$.

10. .Itard,J., 1986, Les glossines ou mouches tsé-tsé, Institut d'élevage et de Médecine vétérinaire des pays tropicaux (IEMVT), , 155pp.

11. Itard J., 1968, Enquête entomologique dans la région des savanes (République du Togo). Revue d'Elevage et de Méd. Vét. Pays Tropicaux, 21(3), 375-368

12. ITRA, 2005, Production animale au Togo;Situation de référence 128 p.

13. ITRA, 2008, 2009,2010, Rapport d'activité

14. Leforban Y., 1994, Etude de la prévalence des infections à trypanosomes chez différentes espèces d'animaux sauvages du parc national de la Comoe en Côte d'Ivoire: résultats préliminaires sur la comparaison de trois méthodes de diagnostic, Revue Elev. Med. vet. Pays trop., 47 (2), 189-194.

15. Leak S.G.A.,Awoume K.,Collardelle C., Duffera w.,Ferron A., 1987, Détermination de la pression glossinaire et ses relations avec la prévalence des trypanosomes dans le bétail trypanotolérant dans les sites du Réseau africain d'étude du bétail trypanotolérant. In Production animale dans les régions d'Afrique infestée par les glossines. Nairobi (Kenya), CIPEA/ILRAD 1988. 
16. Lekeux M., 2006, Trypanosomose bovine africaine : généralité et situation au Bénin. Thèse de Doctorat vétérinaire ; Université ClaudeBernard-Lyon 1

17. Macleannan,K.J.R., 1981, Incidence de la trypanosomiase par la mouche tsé-tsé sur l'économie rurale en Afrique, deuxième partie : Méthodes de lutte ou d'éradication employées contre les infestations par les tsé-tsé, Revue mondiale zootechnie, ,37,9-19

18. Mainguet,J.M., 2000,Thèse pour obtenir le grade de docteur vétérinaire ; soutenue à la faculté de Médecine de Créteil, , 262p.

19. Mawuena K., 1980, Glossina tachinoïdes au sud du Togo et distribution de glossine à Avétonou. Trypanotolérance et production animale, no 1, pp 1-8

20. Mawuena K., Mensan K., 1981, Distribution des glossines au sud du Togo, Trypanotaulérence et Production Animale. Publication No.2

21. Mawuena K., Yacnambe S.1987, Utilisation des pièges à écrans imprégnés d'insecticide pour la lutte contre la trypanosomiase animale. Trypanotolérence et production animale No5

22. Megalie L., 2006, Trypanosomose bovine africaine: Généralités et situation au Bénin; Thèse de doctorat en médecine vétérinaire ; Université de Claude-Bernard -Lyon I,; 89p

23. Mihok, S.,Maramba O.,Munyuki,E., 1995, Mecanical transmission of trypanosome spp. By african stomoxynae (Diptera : Muscidae), Trop. Med. Parasitol., 46,103-106.

24. Mihok, S., 2002, The development of a multipurpose trap (the N'zi ) for tsetse and other biting, Bull. Entomol. Research, 2002, 92 (5) 385403

25. Murray M., Dexter T.M., 1988, Anaemia in bovine african trypanosomiasis. Acta Tropica, $45: 389-432$

26. Murray M., Trail J.C.M. and d'Ieteren G.D.M., 1990, Trypanotolerant in cattle and prospect of the controls of the trypanosomiasis by selecting breeding. Rev. Sci. Tech. OIE 9 : 369-386.

27. Peregrine A.S., 1994, Chemotherapy and delivery système : haemoparasites. Veterynary Parasitology. 54 : 223-248

28. Rickenback A.,1961 Carte de répartition des glossines en afrique occidentale d'expression francaise, ORSTOM, 1961. Trypanotolérance et production animale No1, p.60

29. Swallow B. M., 2000, Impact of trypanosomiasis on African Agriculturre. PAAT Technical and scientific series : 2. FAO, Rome, Italy, $52 \mathrm{p}$

30. Talaki E.,Dao B., Kulo A.,N'feidé T., 2013, Enquête entomologique et parasitologique sur les trypanosomoses bovines à la station d'Avétonou/ ITRA au Togo ; Bull. Anim. Hlth. Prod. Afr., 61 33-38. 
31. Talaki E. Dao B., Dayo T.,N'feidé T., Soedji K., 2014, Etudes entomologiques et parasitologiques des trypanosomoses bovines dans la prefecture de vo au sud du Togo. In : Revue Africaine de Santé et de Production animales ; EISMV de Dakar ; 12,1, 1-30.

32. Touré S.M., 1977, La trypanotolérance. Revue de connaissances. Rev. Elev. Med. Vet. Pays trop. 1977, 30 (2) 157-174 